\title{
Caracterizando controversias sociocientíficas en la prensa escrita. Una herramienta para el desarrollo de la alfabetización científica
}

\author{
Naira Díaz Moreno \\ Departamento de Didáctica de las Ciencias Experimentales. Universidad de Almería. España. \\ naira.diaz@gmail.com \\ ORCID: https:// orcid.org/0000-0002-6983-9141
}

[Recibido: 18 Mayo 2017. Revisado: 27 Enero 2018. Aceptado: 16 Octubre 2018]

\begin{abstract}
Resumen: Dentro de la Enseñanza de las Ciencias, la alfabetización científica constituye un objetivo prioritario, tal y como aparece reflejado en los últimos currícula internacionales. El uso de la prensa con controversias sociocientíficas constituye una buena herramienta para trabajar la alfabetización en el aula de ciencias, siendo para ello necesario obtener unos criterios de selección de noticias controvertidas. Este estudio desarrolla indicadores de controversias sociocientíficas a través de una metodología mixta: cuantitativa y cualitativa. Los posibles indicadores fueron aplicados a una temática controvertida local, el tema del agua en Almería (España), con la finalidad de verificar si la temática constituye o no una controversia sociocientífica. Para ello, se seleccionaron dos muestras comparables de noticias correspondientes a un periodo normal y a un periodo controvertido de nuestro tópico. Los resultados nos han aportado cuatro indicadores de controversias sociocientíficas que pueden ser fácilmente extrapolados a otras temáticas controvertidas que aparezcan en la prensa local y que nos permiten estudiar estos problemas relacionados con la Ciencia socialmente relevantes que afectan y rodean a la ciudadanía.
\end{abstract}

Palabras clave: Alfabetización científica; Controversias Sociocientíficas; Prensa.

\section{Portraying Socioscientific Issues in the Press. An Educational Tool for Scientific Literacy}

Scientific literacy is a major goal in Science Education as it has been reflected in recent international curricula. Using the press with socioscientific news is a suitable tool to develop scientific literacy in the science classroom and for this reason some criteria for the selection of socioscientific news are required. This study seeks to develop socioscientific issues indicators using mixed methods that could be use for teachers as a tool to develop scientific literacy in the classroom. The possible indicators of SSI were applied to a local controversial topic, the subject of water in Almeria (Spain) in order to verify whether this subject is treated as a SSI in the press. About data collection, two sample of news were selected by different approaches including news from a controversial and a noncontroversial period. The main results are four indicators to identify socioscientific issues in the press that could also be applied to different controversial subjects that appear in the local press for their later use in the science classroom. These press issues will allow us to study some socially relevant problems related to science that affect and surround the citizenship.

Keywords: Scientific Literacy; Socioscientific Issues; Press.

Para citar este artículo: Díaz Moreno, N. (2019) Caracterizando controversias sociocientíficas en la prensa escrita. Una herramienta para el desarrollo de la alfabetización cinetífica. Revista Eureka sobre Enseñanza y Divulgación de las Ciencias 16 (1), 1102. doi:10.25267/Rev_Eureka_ensen_divulg_cienc.2019.v16.11.1102

\section{Introducción y justificación del estudio}

Uno de los grandes objetivos de la Enseñanza de las Ciencias ha sido y es alcanzar una población científicamente alfabetizada capaz de tomar decisiones informadas (AAAS, 2008; NRC, 1996), dado que la sociedad se enfrenta cada vez con mayor frecuencia a diferentes cuestiones relacionadas con la Ciencia. Sin embargo, y a pesar de que la comunidad de la Enseñanza de las Ciencias está unida en esta llamada para mejorar la alfabetización científica, no existe un consenso claro en cuánto qué la caracteriza. Roberts (2007) proporciona un heurístico para ello y señala distintas visiones de la alfabetización científica. En este estudio 
asumimos la visión II de Roberts, que sugiere que los individuos científicamente alfabetizados deben de ser capaces de enfrentar, negociar y tomar decisiones en situaciones de la vida diaria que están relacionadas con la ciencia, también conocidas como controversias sociocientíficas (Sadler, 2011).

En esta línea, distintos currícula internacionales destacan la importancia de los contextos que utilizamos para enseñar ciencia y sobre la Ciencia, los cuales se deben de enmarcar dentro de las temáticas sociales y personales que afectan y rodean a los ciudadanos con la finalidad de capacitarlos para tomar decisiones informadas y que participen y contribuyan positivamente con la sociedad (Fowler y Zeidler, 2016). Coincidimos con Sadler (2011) y consideramos que las controversias sociocientíficas nos aportan estos contextos de aprendizaje donde los estudiantes realmente se enfrentan a cuestiones sociales importantes relacionadas con la ciencia y adquieren experiencias negociando sus complejidades. Es en este ámbito de cuestiones científicas emergentes y no consensuadas relacionadas con la sociedad donde desarrollamos nuestra investigación.

Para situar las controversias sociocientíficas tomamos la definición de Sadler (2004) que nos dice que las controversias sociocientíficas, en inglés socioscientific issues (SSI) y en adelante CSC, son cuestiones sociales controvertidas con vínculos conceptuales y/o de procedimiento a la ciencia. Son además complejas, abiertas y están formadas por problemas los cuales carecen de soluciones simples y directas. Las posibles soluciones de estas cuestiones pueden ser informadas por principios científicos, teorías y datos, pero además, están influenciadas por distintos factores sociales (Sadler, 2011). Las CSC han sido promovidas por diversos autores como herramientas pedagógicas para enseñar ciencia, fomentar la alfabetización y el carácter científico funcional para los ciudadanos globales (e.g. Zeidler, Berkowitz y Bennett, 2013). En esta línea, Zeidler (2014) alude a ellas como puntos de entrada en el currículo de ciencias que permiten cultivar la alfabetización científica promoviendo el razonamiento informal.

Por otro lado, hemos de señalar que la alfabetización científica está conectada con los medios de comunicación puesto que ser capaz de tratar con la Ciencia en las noticias está considerado una demostración de alfabetización científica (Zimmerman et al., 1999). Consecuentemente, una educación basada en el refuerzo de esta alfabetización debe cruzarse, de alguna manera, además de con las CSC, con la Ciencia en las noticias (NRC, 1996); puesto que estar alfabetizado científicamente significa tanto ser capaz de leer de una manera entendible la ciencia que aparece en las noticias de prensa como participar en una discusión acerca de la validez de sus conclusiones (Jarman y McClune, 2007). Como ya hemos señalado, la sociedad ha de tomar decisiones sobre CSC que en ocasiones son recogidas por la prensa (McClune y Jarman, 2014), que ejerce como principal canal divulgador de noticias científicas y que, por tanto, puede ser generadora de CSC convirtiéndose en una herramienta didáctica muy útil para trabajar la alfabetización científica (Feinstein, 2015), lo que nos hizo plantearnos cómo podíamos determinar noticias con CSC para su posterior uso en el aula de ciencias.

En la literatura hemos encontrado estudios de prensa donde se analiza el contenido de distintas temáticas controvertidas como el cambio climático (e.g. Poberezshkaya, 2015; Bowe et al., 2014); también existen investigaciones acerca del carácter consensuado que presentan las noticias científicas (e.g. Vílchez, 2009). Sin embargo, no hemos encontrado investigación alguna que especifique cómo se ha analizado la controversia o qué indicadores se han aplicado para afirmar que una noticia es o no controvertida, por lo que podemos decir que existe un vacío respecto a cómo determinar CSC, justificando así la necesidad de nuestra investigación.

El objetivo general de la presente investigación se pueden desglosar en: 1) identificar indicadores de CSC en prensa escrita; y 2) aplicar esos indicadores a un tópico concreto (agua) en prensa local que nos permitan valorar cuándo una temática constituye una CSC. 
Hemos seleccionado, para aplicar los indicadores, una problemática local por la proximidad que tiene al alumnado, así como por la disponibilidad del recurso, puesto que los periódicos locales suelen encontrarse en todos los centros escolares. La temática de estudio fue escogida por diferentes razones: por un lado, el agua ha sido y sigue siendo un contenido escolar presente en diferentes propuestas curriculares. Las últimas propuestas educativas están abordando diversos aspectos relacionados con la importancia del agua para los seres vivos y su agotamiento como recurso, planteando problemas socialmente relevantes relacionados con su uso y gestión (Fernández-Arroyo y Rodríguez-Marín, 2016). Por otro, Almería, que es donde se desarrolla nuestra investigación, está localizada en el extremo sureste del país y se caracteriza por su aridez geográfica, siendo una de las provincias con menor índice pluviométrico de Europa, lo que explica su escasez de recursos hídricos superficiales que coexiste con una alta demanda hídrica por parte de la agricultura. Además, el tema del agua es percibido como uno de los principales problemas ambientales, tal y como se puso de manifiesto en el debate creado en 2004 con la derogación del Plan Hidrológico Nacional (PHN) y del trasvase del río Ebro, el más caudaloso de España, para transferir agua desde el extremo noreste hasta el sureste del país (Valencia, Alicante, Murcia y Almería) que provocó una fuerte polémica a nivel nacional por el coste económico y el daño medioambiental que produciría el trasvase. Como apuntan Aguerri y Bravo-Torija (2017) los ríos son sistemas naturales complejos cuya gestión es motivo de polémica que aparece de forma recurrente en los medios de comunicación. Estos hechos, junto con la implantación de la Directiva Europea Marco Agua en el 2000, nos llevaron a pensar que esta temática podría ser una CSC que aparece reflejada en la prensa local, y nos puede servir de caso para identificar los indicadores de controversias.

\section{Metodología}

Para estudiar las noticias controvertidas en prensa y llevar a cabo la búsqueda de indicadores que nos ayuden a determinar si un tópico constituye una CSC hemos empleado una metodología mixta, cuantitativa y cualitativa, puesto que una combinación de ambos enfoques puede ser una forma particularmente prometedora de hacer investigación (Schäfer, 2012). Dentro del análisis cuantitativo, llevamos a cabo una revisión bibliográfica que nos permitió determinar las principales temáticas controvertidas estudiadas tanto en el ámbito educativo como en el periodístico, así como las propuestas educativas existentes (Díaz-Moreno y Jiménez-Liso, 2012). El posterior análisis documental nos proporcionó categorías que utilizamos como posibles indicadores de CSC a los que llamamos CSC-1: presencia, importancia de la materia presentada en las noticias, tipo de evento que presentan las noticias, áreas de la vida más influidas por la noticia y sub-temas tecnocientificos frecuentes y presencia de corrientes sociocientificas en las noticias y que aplicamos a un tema concreto, el agua, con la finalidad de determinar su utilidad como indicadores de CSC y si el tema del agua constituye una CSC. Para la toma de muestra se realizaron dos aproximaciones distintas: "semana construida" (Dimopoulos y Koulaidis, 2003) y "noticia-testigo" (Fernández-Muerza, 2004) que nos permitieron obtener dos muestras de noticias comparables. La primera muestra, a la que denominamos normal, está formada por 50 noticias sobre el agua tomadas de los dos periódicos locales de mayor relevancia (La Voz de Almería e Ideal). Para su selección se han realizado catas de semanas completas durante los meses de junio, febrero y octubre desde el año 1992 hasta 2008. La segunda muestra la obtuvimos aplicando la metodología "noticia-testigo", estudiando la línea del tiempo de noticias sobre el agua desde 1992 hasta 2011 (fecha próxima al comienzo de la investigación) y analizando su frecuencia. Determinamos el pico de máxima presencia de noticias en los periódicos nacionales de referencia (El País, La Vanguardia y El Mundo), obteniendo como resultado marzo de 2004. Posteriormente seleccionamos las noticias que fueron publicadas en 
marzo de 2004 en la prensa local, obteniendo una segunda muestra de 69 noticias a la que llamamos muestra en estado controvertido.

La metodología cualitativa, que hemos desarrollado de manera emergente y por saturación, es la llamada Cartografía de las Controversias, que surge de la Teoría del Actor Red (Latour, 2005). Esta teoría nos ha aportado el esquema conceptual idóneo para poder estudiar las relaciones que se establecen en estos asuntos controvertidos relacionados con la Ciencia y la Tecnología. Siguiendo a Alcíbar (2007), en la construcción de hechos científicos intervienen factores sociales y epistémicos, produciéndose complejos procesos de negociación destinados a tener éxito sólo si se logra involucrar a una cada vez más amplia red de actores. Este marco teórico de referencia nos ha permitido observar cómo los diversos actores sociales negocian y exponen sus intereses divergentes para fijar en la trama común determinados aspectos de la controversia. Sobre esta base teórica se constituye la Cartografía de la Controversia, que nos ha permitido estudiar los procesos sociales que tienen lugar en las controversias sociocientíficas y describir los debates formados en torno a ellas. En concreto, nos centramos en los procesos que tienen lugar durante el período que determinamos controvertido o con mayor presencia de noticias, aplicando esta metodología en las 69 noticias de la muestra controvertida y que nosotros representamos utilizando el software Ucinet (Borgatti et al., 2002).

La cartografía de la controversia, por tanto, nos ha permitido determinar dos indicadores de CSC que denominamos CSC-2: Redes de actores y Vinculos entre redes de actores y tipos de evento/corrientes sociocientificas.

\section{Indicadores de prensa CSC-1}

Estos primeros indicadores de CSC fueron extraídos del análisis documental donde seleccionamos distintos estudios (contenido, tratamiento etc.) de noticias científicas en prensa realizados en investigaciones previas (Dimopoulos y Koulaidis, 2003; Jarman y McClune, 2007). Para cada uno de ellos presentamos una justificación acerca de su elección como indicador de CSC, elaboramos una hipótesis sobre los resultados que tendría que mostrar para confirmar la presencia de CSC y lo aplicamos a la temática local seleccionada. De esta manera, determinamos qué indicadores nos aportan más información y si estos caracterizan la CSC por sí mismos o es necesario complementarlos con otros indicadores. Por último, la aplicación de estos indicadores a nuestra temática local nos permite determinar si constituye una controversia sociocientífica tal y como hemos establecido en la introducción.

\section{Presencia}

Los medios de comunicación han sido y siguen siendo el principal canal que conecta la Ciencia con el público en general (Schäfer, 2012). Por tanto, la presencia en prensa de una temática sociocientífica, además de una característica, puede ser un primer indicador de CSC, lo que nos llevó a formular nuestra primera hipótesis, (H1): La presencia puede ser un indicador de controversia sociocientífica puesto que para que haya controversia tiene que haber presencia no anecdótica de noticias científicas.

Para contrastar esta hipótesis no bastaría con dar el cómputo global de una muestra de noticias relacionadas con el tópico agua en los diarios locales, puesto que no nos proporcionaría ninguna evidencia de si la presencia es o no anecdótica. Por ello, hemos seleccionado dos muestras con metodologías diferentes pero con la finalidad de que sean comparables tal y como se detalla en el epígrafe anterior. Para la selección de nuestra primera muestra hemos utilizado, como ya hemos señalado, la metodología "semana construida" (Dimopoulos y Koulaidis, 2003) tomando noticias de los dos periódicos locales de mayor relevancia: La Voz de Almería e Ideal. Seleccionamos la última semana completa de Febrero, 
Junio y Octubre con catas de cuatro años (1992, 1996, 2000, 2004, 2008) que nos permitió obtener una muestra de 50 noticias relacionadas con el tópico del agua durante un periodo prolongado en el tiempo que no estuviese afectado por ningún evento concreto, obteniendo una primera muestra que denominamos normal. La segunda muestra la obtuvimos aplicando la metodología de la "noticia-testigo" (Fernández-Muerza, 2004), a través de la cual analizamos la frecuencia de noticias sobre el tópico agua estudiando la línea del tiempo desde 1992 hasta 2011 (fecha próxima al comienzo de la investigación). De esta manera, determinamos el año en que se produjo un mayor aumento de noticias sobre esta temática en los periódicos nacionales de referencia (El País, La Vanguardia y El Mundo), obteniendo como resultado el año 2004. Una vez detectado este pico, realizamos la línea del tiempo mensual obteniendo un máximo de noticias en el mes de marzo, por lo que tomamos esta fecha, marzo de 2004, para seleccionar todas las noticias publicadas en la prensa almeriense sobre el tópico agua. 69 noticias, a modo de "mes construido", constituyeron la muestra en estado controvertido comparable a la muestra normal seleccionada anteriormente.

Los resultados de presencia para la muestra en estado normal sobre el tema del agua en Almería, muestran un promedio de 0,47 artículos diarios. Es un promedio bajo si lo comparamos con el obtenido para el tópico Energía Solar en la misma muestra de la prensa almeriense (0,7 artículos diarios, Jiménez-Liso et al., 2010). Sin embargo, para la muestra en estado controvertido, detectamos una abundante presencia del tema del agua, con un promedio de 2,39 artículos diarios. Según estos resultados, la muestra normal presenta escasa presencia en comparación con la muestra controvertida, la cual quintuplica la frecuencia normal. No obstante, la presencia constituye casi una condición previa para la existencia de controversia, y a pesar de haber obtenido resultados antagónicos para ambas muestras, este indicador no nos aporta información suficiente para determinar si nuestra temática es una CSC; precisando por ello, de más indicadores con lo que poder determinar la controversia. Aún así, este indicador sí nos permite detectar periodos controvertidos (Autora, 2016).

\section{Importancia de la materia presentada en las noticias}

Una vez detectada la presencia de las noticias, indagamos la importancia que le concede la prensa a estas noticias y que constituye nuestro segundo indicador. Jarman y McClune (2007) ya destacaron la relevancia como uno de los criterios de selección de noticias, señalando que los acontecimientos que se perciben como influyentes en la vida cotidiana de la audiencia se consideran más noticiables que los que no se perciben así, es decir, las noticias pueden ser de interés o también importantes. Para determinar este indicador adaptamos el protocolo de análisis de noticias de Dimopoulos y Koulaidis (2003) y consideramos una serie de variables que definen la importancia de la noticia: área total de la noticia $\left(\mathrm{cm}^{2}\right)$, tamaño promedio $\left(\mathrm{cm}^{2}\right)$, presencia de imágenes, referencia en portada, ubicación en página interna frontal y sección del periódico. De acuerdo a estas variables enunciamos nuestra segunda hipótesis (H2) : Para que el tópico agua sea una CSC, el indicador importancia debe señalar que las noticias ocupan posiciones preferenciales en la prensa, como son presencia en portada, tamaño grande, presencia de imágenes, etc.

Como resultado, obtuvimos un tamaño promedio sorprendentemente bajo para las noticias de la muestra controvertida, pasando de $366,48 \mathrm{~cm}^{2}$ de tamaño para la muestra en estado normal a $254,84 \mathrm{~cm}^{2}$ en la controvertida. También, es de señalar la similitud en los resultados para ambas muestras de "presencia de imágenes" con los resultados de 64 y 63,77 \%. Sólo las variables "presencia en portada" y "presencia en página frontal" parecen indicar valores más elevados para la muestra controvertida, mostrando valores de un $23,2 \%$ frente a un $20 \%$ en la muestra normal para presencia en portada y 55,1\% frente $42 \%$ para página frontal. Al analizar la importancia de las noticias en función de estas variables, comprobamos que no existen grandes 
diferencias entre ambas muestras y, por tanto, el indicador importancia por sí mismo no parece ser determinante y necesita ser complementado otros indicadores de CSC.

\section{Tipo de evento que presentan las noticias}

Siguiendo a Thompson (1995), la prensa proporciona un reflejo bastante representativo de los procesos sociales de la vida pública. En este sentido, nos interesa averiguar los tipos de evento que presentan las noticias para poder determinar en qué contextos tienen lugar y explorar si constituyen una CSC. Siguiendo a Dimopoulos y Koulaidis (2003) consideramos cinco tipos de eventos (Tabla 1). Tras esta distinción, formulamos nuestra hipótesis (H3): el tópico estudiado constituye una CSC si las noticias se presentan en tipos de evento desarrollados en contextos conflictivos como "política", donde el principal evento reportado relaciona directamente a la actividad científica con aspectos políticos y/o económicos, reflejando el carácter controvertido de la noticia en lugar de en otros tipos de eventos más neutrales como "innovación" o hechos "divulgativos".

Los resultados obtenidos (Tabla 1) señalan que la muestra en estado normal presenta dispersión en los tipos de evento, mientras que la muestra en estado controvertido presenta concentración de eventos de tipo "político-económico" (85,5\%), confirmado nuestra hipótesis e indicando la presencia de controversia con respecto a la temática del agua.

Tabla 1. Resultados relativos a tipo de evento en la muestra normal y controvertida

\begin{tabular}{|lll|}
\hline Tipo de evento & Resultados muestra normal (\%) & Resultados muestra controvertida $(\%)$ \\
\hline Evento político-económico & 40 & 85,5 \\
Evento o contenido divulgativo & 28 & 0 \\
Eventos negativos & 14 & 0 \\
Innovación/Investigación & 12 & 14,5 \\
Evento político-investigación & 6 & 0 \\
TOTAL & 100 & 100 \\
\hline
\end{tabular}

\section{Áreas de la vida más influidas por las noticias}

Para determinar CSC hemos de tener en cuenta, además del contexto, qué interacciones producen la Ciencia y la Tecnología en la vida pública como áreas públicas relacionadas con la noticia, con la finalidad de caracterizar y analizar su contenido. Igual que en indicadores anteriores, seguimos el estudio de Dimopoulos y Koulaidis (2003) que señalan distintas áreas de influencia de las noticias científicas. Enunciamos nuestra cuarta hipótesis (H4): para que la temática de la noticia constituya una CSC el indicador áreas de la vida, debe señalar que existe interacción entre diferentes áreas, fundamentalmente entre aquellas relacionadas con políticos/as, científicos/as y economía (situadas en nuestro análisis dentro del área "administración”). En este sentido, si existe controversia, esta provocará distintas opiniones en áreas diferentes mostrando la interacción entre diversas áreas sociales.

Los resultados obtenidos (Tabla 2) muestran que para la muestra normal las áreas mayoritarias son aquellas relacionadas con "campo" y "servicios", mientras que para la muestra controvertida es mayoritaria el área "administración" con un 55,07\%, en la que se incluyen las noticias relacionadas con "política" y "economía", áreas más afectadas por las controversias. Los resultados relativos al área "campo" permanecen estables tanto en la muestra normal como controvertida, lo que parece indicar que el periódico mantiene su carácter agrario (principal sector de la economía almeriense y, por tanto, público receptor) aunque se esté generando una controversia. 
Tabla 2. Resultados obtenidos en áreas de la vida para la muestra normal y controvertida

\begin{tabular}{lcc}
\hline $\begin{array}{l}\text { Áreas de la vida más } \\
\text { influidas }\end{array}$ & Resultado de la muestra normal (\%) & Resultado de la muestra controvertida (\%) \\
\hline Del campo & 44 & 34,21 \\
De los servicios & 36 & 7,25 \\
De la investigación & 8 & 8,69 \\
De la administración & 8 & 55,07 \\
De la salud & 4 & 7,25 \\
De la enseñanza & 0 & 0 \\
De las humanidades & 0 & 0 \\
De la protección social & 0 & 0 \\
Jurídica & 0 & 2,89 \\
TOTAL & 100 & 100 \\
\hline
\end{tabular}

Las diferencias de resultados obtenidos para ambas muestras nos permiten afirmar que el indicador áreas de la vida aporta suficiente información por sí mismo para determinar CSC.

\section{Sub-temas tecnocientíficos frecuentes y presencia de corrientes sociocientíficas en las noticias}

La prensa juega un papel fundamental a la hora de aportar información a la opinión pública sobre temas relacionados con la Ciencia y Tecnología (Dimpoulos y Koulaidis, 2002), por eso es de especial interés analizar qué temáticas y contenidos relacionados con la Ciencia son los que aparecen en las noticias y si lo hacen de manera controvertida. En este indicador estudiamos los contenidos relacionados con el tema que tratamos agrupándolos por temáticas, lo que nos permitirá conocer qué sub-temas son los más repetidos y si estos contenidos se presentan o no con un enfoque controvertido, lo que hemos llamado corrientes sociocientíficas; diferenciando así si el enfoque de la noticia también es controvertido, independientemente de la temática. Enunciamos la hipótesis (H5): el tópico estudiado constituye una CSC si el indicador señala que existe gran concentración de sub-temas, y que éstos se plantean bajo un enfoque controvertido (corrientes sociocientificas).

Para determinar sub-temas y corrientes sociocientificas hemos utilizado un agrupamiento por similitud y categorizado de manera emergente. Los resultados de la muestra normal indican la dispersión de sub-temas $(\mathrm{n}=12)$ entre los que destacan por su mayor frecuencia "obras hidráulicas" ( $\mathrm{n}=6)$ y "depuración/desalación de agua" ( $\mathrm{n}=4)$ mientras que para la muestra controvertida determinamos una mayor concentración de sub-temas (7), siendo mayoritarios "depuración y desalación de agua" y "gestión de agua". Algo similar sucede para corrientes sociocientificas: en la muestra normal determinamos 10 corrientes sociocientificas que se concentran en 5 para la muestra controvertida (déficit hídrico, PHN, sobreexplotación de recursos hídricos, eficiencia hídrica y despilfarro hídrico). Los resultados, por tanto, aluden a una dispersión de sub-temas y baja presencia de corrientes sociocientificas en la muestra normal, mientras que la muestra controvertida presenta alta concentración de sub-temas y de corrientes sociocientificas, por consiguiente, el indicador señala que el tema del agua constituye una CSC.

\section{Indicadores de prensa CSC-2}

\section{Actores y Redes de actores}

Un actor según la "cartografía de la controversia" es cualquier cosa que hace algo: seres humanos, objetos técnicos, organismos, etc. (Venturini, 2010). Siguiendo las premisas 
establecidas por esta metodología determinamos los distintos actores que intervienen en la problemática del agua anotando cada actor que aparecía en las noticias seleccionadas de la muestra controvertida y agrupándolos por similitud para establecer categorías de actores (nivel 1). Posteriormente, redes de actores, que sería el nivel máximo de agrupación (nivel 2) y que revelan los distintos discursos existentes. Establecimos nuestra hipótesis señalando que (H6): el tópico estudiado constituye una CSC si existe dispersión en las redes de actores que participan en la temática, lo cual señala que ésta afecta a un gran número de actores con diversos intereses y, por tanto, es controvertida.

Como resultados hemos obtenido un total de 17 redes de actores que surgen de la agrupación de más de 30 categorías de actores. Organismos públicos, Política, Organismos privados, Regiones o provincias implicadas y Desalación son las redes que aparecen con mayor frecuencia en la temática del agua, con una frecuencia superior a 20. La amplia dispersión de redes de actores y la heterogeneidad de las mismas pone de manifiesto que nuestro tópico constituye una CSC.

\section{Vínculos establecidos entre redes de actores, tipos de evento y corrientes sociocientíficas}

Este indicador pone en relación las redes de actores con dos indicadores CSC-1, que nos permitirá establecer los vínculos que se forman entre redes de actores, contextos y contenidos de la problemática a modo de mapa. En este caso nuestra hipótesis (H7) es: los vínculos redes de actores, tipos de evento y corrientes sociocientíficas indicarán la existencia de controversia si nos muestran, por una parte, que las relaciones se establecen entre redes de actores y tipos de eventos controvertidos permiten describir qué tipo de evento genera más controversia para un tema determinado. Por otra parte, los vínculos redes de actores-corrientes sociocientíficas permitirán mostrar qué corrientes son las que generan más controversia por la concentración de redes de actores con las que se relaciona. Para mostrar los resultados de este indicador realizamos dos mapas donde hemos representado los vínculos establecidos entre redes de actores y tipos de evento (Figura 1) y vinculos establecidos entre redes de actores y corrientes sociocientificas (Figura 2).

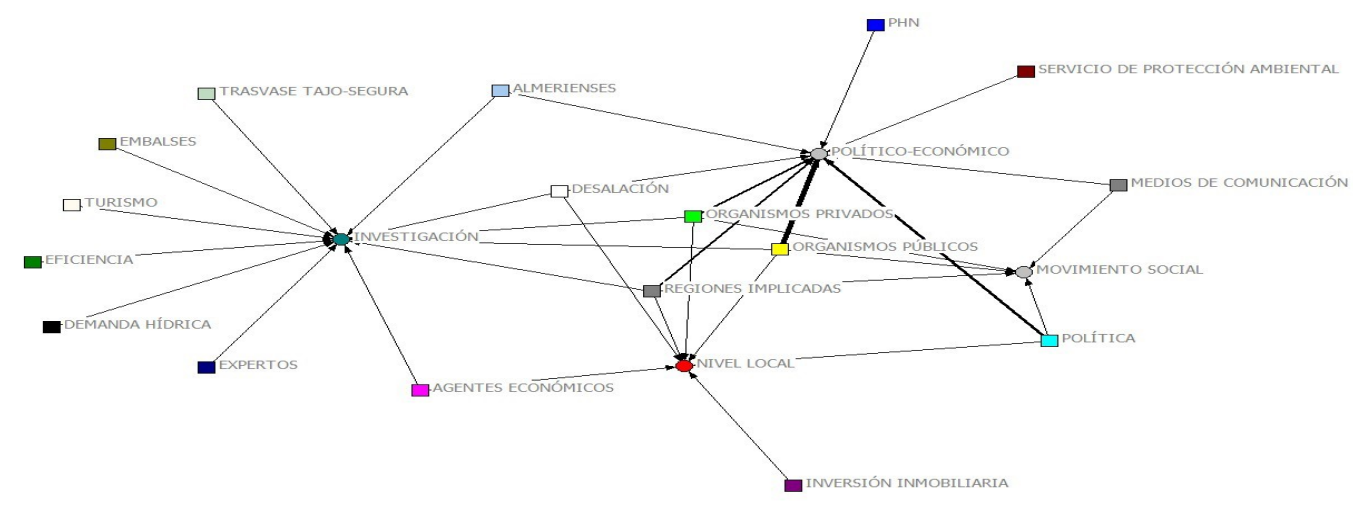

Figura 1. Representación del mapa de vínculos entre redes de actores y tipos de evento

Obtuvimos un primer mapa (Figura 1) donde aparecen representados las 17 redes de actores detectadas(cuadrados) así como los 4 tipos de eventos (círculos) determinados en las noticias de la muestra controvertida. Las redes de actores que presentan más vínculos son "Organismos públicos", "Organismos privados" y "Regiones implicadas", apareciendo mayoritariamente en los contextos "político-económico" o aquellos relacionados con la "investigación". 
Por otra parte, los vinculos establecidos entre redes de actores y corrientes sociocientificas los representamos en la figura 2 donde se recogen las 17 redes de actores (cuadrados) y, en este caso, las 5 corrientes sociocientificas (círculos) detectados en la muestra controvertida.

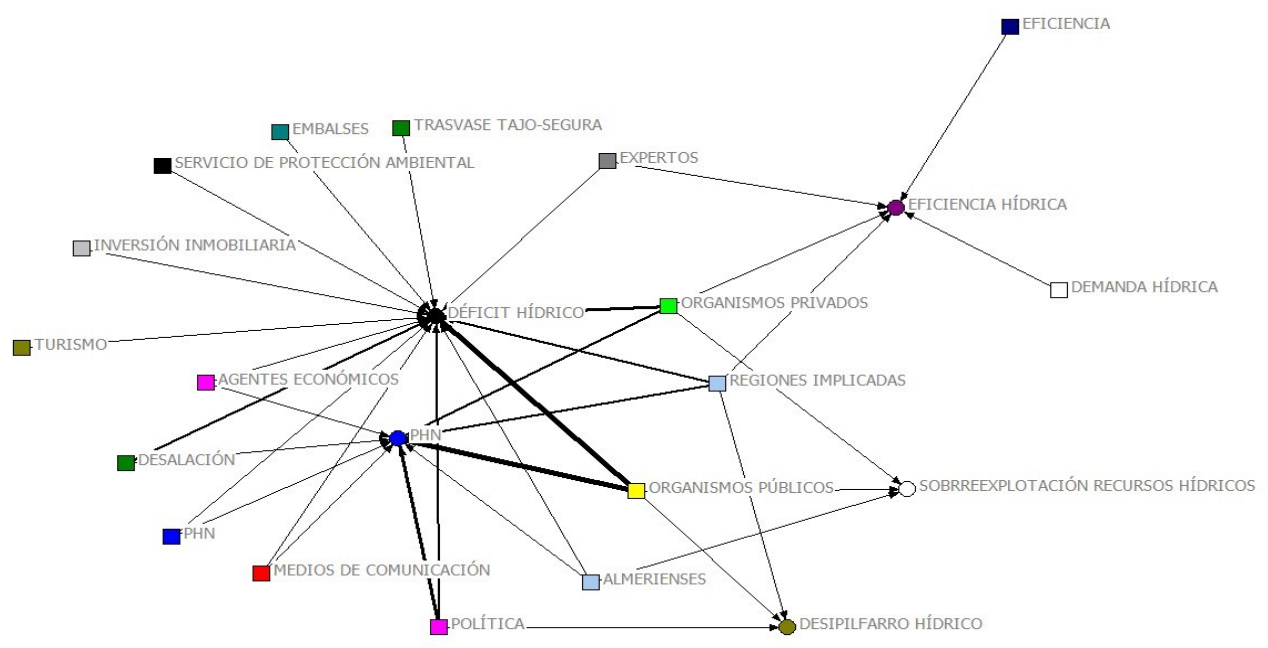

Figura 2. Representación del mapa de vínculos entre redes de actores y corrientes sociocientíficas

Igual que en la figura 1, las redes de actores que aparecen con mayor número de vínculos con las corrientes sociocientíficas (4 o más) son "Organismos públicos", "Organismos privados" y "Regiones implicadas" y todas ellas tienen en común su conexión con "PHN" o "déficit hídrico". Por tanto, podemos decir que estas dos corrientes sociocientificas están vinculadas a la mayoría de las redes de actores (15 y 9 vínculos respectivamente) y son las que generan más controversia en la temática del agua. Los vínculos nos permiten obtener una visión amplia de la CSC donde se muestran los diferentes intereses señalando su naturaleza real tal y como aparece reflejada en la prensa, así como determinar en qué contextos y qué contenidos son los más relacionados con la problemática estudiada, convirtiéndose en una herramienta excelente para describir en profundidad las CSC. Para la temática del agua era de esperar que uno de los nodos de mayor controversia fuera el PHN, ya que las noticias seleccionadas durante el período controvertido corresponden con el período de máxima controversia a nivel de prensa nacional (marzo de 2004). No obstante, aparecen dos corrientes "autóctonas": "déficit hídrico" y "eficiencia hídrica", que constituyen dos concepciones alternativas bien arraigadas en la población, relacionadas con la baja pluviometría de la provincia, y que contrastan con el interés económico-comercial para la agricultura intensiva.

\section{Resultados}

Los primeros indicadores seleccionados (CSC-1) han sido aplicados al tópico del agua en dos muestras comparables de noticias, tomadas de la prensa almeriense, correspondientes a un periodo normal y otro controvertido con la finalidad de valorar si sirven para identificar CSC. Así, y dado que conocemos que el tema del agua constituye una CSC a nivel nacional durante el período controvertido, hemos establecido que nuestras hipótesis se cumplen cuando se verifican para la muestra en este periodo. Por otro lado, y en función de la información que aporta cada indicador, los hemos clasificado en indicadores generales y específicos. Los indicadores generales son aquellos en los que la hipótesis no se cumple para la muestra controvertida o que no aportan suficiente información por sí mismos para determinar CSC y necesitarán ser triangulados con otros indicadores para poder valorar si un tópico constituye o no una controversia. Por otro lado, los indicadores específicos, son aquellos que corroboran la 
hipótesis de partida en la muestra controvertida, determinan de forma clara la existencia de CSC y permiten caracterizarlas por sí mismos. En las tablas 3 y 4 mostramos un resumen de las hipótesis planteadas para cada indicador, resultados obtenidos tras haberlos aplicado en nuestra temática y destacando aquellos resultados obtenidos en la muestra controvertida. Por último, resultados como indicador y clasificación de cada uno de ellos.

Tabla 3. Resultados obtenidos tras aplicar los indicadores CSC-1

\begin{tabular}{|c|c|c|}
\hline Hipótesis & Resultados en el tópico agua & Resultados como indicador \\
\hline $\begin{array}{l}\text { H1: La presencia puede ser un } \\
\text { indicador puesto que para que haya } \\
\text { controversia tiene que haber } \\
\text { presencia no anecdótica de noticias. }\end{array}$ & $\begin{array}{l}\text { Se cumple la hipótesis para la } \\
\text { muestra controvertida con abundante } \\
\text { presencia de noticias. Indica que el } \\
\text { agua es una CSC en esa muestra. }\end{array}$ & $\begin{array}{l}\text { La presencia es una condición } \\
\text { indispensable para constituir una } \\
\text { CSC pero no aporta información } \\
\text { suficiente. Indicador general. }\end{array}$ \\
\hline $\begin{array}{l}\text { H2: Para que el tópico agua sea una } \\
\text { CSC, el indicador importancia debe } \\
\text { señalar que las noticias ocupan } \\
\text { posiciones preferenciales en la } \\
\text { prensa. }\end{array}$ & $\begin{array}{l}\text { Solo alguna de las variables del } \\
\text { indicador aparecen en la muestra } \\
\text { controvertida, por lo que la existencia } \\
\text { de controversia es dudosa. No } \\
\text { señala el agua como una CSC. }\end{array}$ & $\begin{array}{l}\text { No aporta información suficiente } \\
\text { para determinar CSC. Indicador } \\
\text { general. }\end{array}$ \\
\hline $\begin{array}{l}\text { H3: el tópico constituye una CSC si } \\
\text { las noticias se presentan en tipos de } \\
\text { evento de contexto conflictivo como } \\
\text { "política". }\end{array}$ & $\begin{array}{l}\text { La muestra controvertida presenta } \\
\text { concentración de eventos de tipo } \\
\text { "político-económico" y nos } \\
\text { confirma la existencia de CSC en el } \\
\text { caso del agua. }\end{array}$ & $\begin{array}{l}\text { Nos permite valorar por sí mismo } \\
\text { cuando un tópico constituye una } \\
\text { CSC. Indicador específico. }\end{array}$ \\
\hline $\begin{array}{l}\text { H4: Para que exista CSC el } \\
\text { indicador áreas de la vida, debe } \\
\text { mostrar interacción entre diferentes } \\
\text { áreas (fundamentalmente con el } \\
\text { área "administración"). }\end{array}$ & $\begin{array}{l}\text { La hipótesis se verifica en la } \\
\text { muestra controvertida donde un } \\
55,07 \% \text { corresponde al área } \\
\text { "administración", situando el agua } \\
\text { como una CSC. }\end{array}$ & $\begin{array}{l}\text { Aporta suficiente información por sí } \\
\text { mismo para determinar CSC. } \\
\text { Indicador específico. }\end{array}$ \\
\hline $\begin{array}{l}\text { H5: el tópico estudiado constituye } \\
\text { una CSC si el indicador señala que } \\
\text { hay gran concentración de sub-temas, } \\
\text { y que éstos se plantean bajo un } \\
\text { enfoque controvertido (corrientes } \\
\text { sociocientificas). }\end{array}$ & $\begin{array}{l}\text { Los resultados en la muestra } \\
\text { controvertida aluden a una alta } \\
\text { concentración de sub-temas y de } \\
\text { corrientes sociocientificas. Según el } \\
\text { indicador en esta muestra el agua es } \\
\text { una CSC. }\end{array}$ & $\begin{array}{l}\text { Los resultados obtenidos nos dan } \\
\text { idea de que el indicador nos permite } \\
\text { valorar la presencia de CSC sin } \\
\text { compararlo con otros indicadores. } \\
\text { Indicador específico. }\end{array}$ \\
\hline
\end{tabular}

Con respecto a los indicadores CSC-2, surgieron como resultado de la búsqueda de información acerca del contexto en el cual se genera el problema que aparece en la noticia. Estos también han sido clasificados como generales, que servirán para triangularlos con los CSC-1, o específicos. Tras aplicarlos al tópico del agua obtuvimos los siguientes resultados:

Tabla 4. Resultados obtenidos tras aplicar los indicadores CSC-2

\begin{tabular}{|c|c|c|}
\hline Hipótesis & Resultados en el tópico agua & Resultados como indicador \\
\hline $\begin{array}{l}\text { H6: el tópico estudiado constituye } \\
\text { una CSC si existe dispersión de } \\
\text { actores que participen en la temática. }\end{array}$ & $\begin{array}{l}\text { En la muestra controvertida se cumple } \\
\text { la hipótesis al determinar una } \\
\text { amplia dispersión de redes. El agua } \\
\text { constituye una CSC en esta muestra. }\end{array}$ & $\begin{array}{l}\text { Aporta información sobre el } \\
\text { contexto de la CSC. Indicador } \\
\text { específico. }\end{array}$ \\
\hline $\begin{array}{l}\text { H7: este indicador muestra } \\
\text { controversia si determinamos } \\
\text { relaciones entre redes de actores y tipos } \\
\text { de eventos controvertidos, así como } \\
\text { entre corrientes y concentración de } \\
\text { actores. }\end{array}$ & $\begin{array}{l}\text { Para la muestra controvertida los } \\
\text { vínculos se establecen de manera } \\
\text { mayoritaria entre tipos de evento } \\
\text { controvertidos y muestran } \\
\text { concentración de actores en torno a } \\
\text { corrientes sociocientíficas, } \\
\text { caracterizando el agua como una } \\
\text { CSC. }\end{array}$ & $\begin{array}{l}\text { Aporta y amplia información sobre } \\
\text { el contexto de la CSC. Indicador } \\
\text { específico. }\end{array}$ \\
\hline
\end{tabular}




\section{Conclusiones}

En este artículo se proponen y estudian distintos indicadores para determinar CSC en prensa escrita aplicándolos a una temática local concreta. Hemos obtenido un total de 7 indicadores y todos ellos han sido aplicados a dos muestras comparables de noticias de la prensa almeriense que se corresponden con un periodo normal y otro controvertido, con la finalidad de valorar si sirven para identificar si el tema del agua constituye una CSC. Tras los resultados obtenidos, los hemos clasificado según sean útiles o no para determinar controversias por sí solos (indicadores específicos) o precisan de otros (indicadores generales), obteniendo un total de 5 indicadores específicos y 2 generales, que precisan de más información para poder determinar cuando existe una CSC.

Con respecto al tema del agua, ninguno de los indicadores CSC-1 detecta presencia de controversia en la muestra normal, mientras que para la muestra controvertida, 4 de ellos indican la existencia de controversia. Para completar esta información, aplicamos a nuestra temática los indicadores CSC-2, y obtuvimos que ambos indicadores señalan la existencia de controversia en la muestra controvertida, por lo que podemos afirmar sin lugar a dudas que en este período el tema del agua constituye un CSC.

Determinar CSC en prensa es importante puesto que, de una parte la selección de noticias con controversias debe ser el paso previo de todo docente que quiera utilizar la prensa en el aula de ciencias (Jarman y McClune, 2007; Bowe et al., 2014). De otra, y como ya hemos señalado, el uso de prensa con CSC permite fomentar desarrollar la alfabetización científica, una de las principales finalidades en la Enseñanza de las Ciencias, utilizando como contexto las CSC que normalmente surgen cuando existe una diferencia de opiniones o desacuerdos entre periodistas, ciudadanos y científicos pero que además están sometidas a otros intereses.

Este contexto de CSC en las noticias es especialmente relevante en la educación científica puesto que pueden servir para que los estudiantes construyan su conocimiento social y que actúen, en palabras de Feinstein (2011), como compentents outsiders (foráneos competentes), personas que reconocen los momentos en los que la Ciencia tienen importancia en sus necesidades e intereses e interaccionando para conseguir sus objetivos. Las noticias con CSC proporcionan una situación atractiva de la Ciencia pero sobretodo, permiten democratizar la formación del conocimiento, como en el caso del dragado del río Ebro (Aguerri y BravoTorija, 2017), ayudando a nuestro estudiantes a desarrollar esquemas intelectuales auto sostenibles en cuestiones que suelen estar monopolizadas por las autoridades o responsables políticos (Zeidler, 2014).

\section{Referencias bibliográficas}

AAAS (American Association for the Advancement of Science). (2008). Exploring the nature of science: Using the Atlas of Science Literacy and other education resources from AAS Project 2061. Recuperado el 3 de Febrero del 2016 de http://www.project2061.org/publications/guides/science.pdf

Aguerri M., Bravo-Torija B. (2017) El uso de pruebas en la resolución de problemas reales en $4^{\circ}$ de ESO:¿ debemos dragar el río Ebro?. Revista Eureka sobre Enseñanza y Divulgación de las Ciencias, 14 (2), 302-316.

Alcíbar M. (2007) Comunicar la ciencia. La clonación como debate periodístico. Madrid: Consejo Superior de Investigaciones Científicas.

Borgatti S. P., Everett M. G., Freeman L. C. (2002) Ucinet for Windows: Software for Social Network Analysis. Harvard, MA: Analytic Technologies. 
Bowe B. J., Oshita T., Terracina-Hartman C., Chao W. (2014) Framing of climate change in newspaper coverage of the East Anglia e-mail scandal. Public Understanding of Science, 23 (2), 157-169.

Feinstein N. (2015) Education, Communication and Science in the Public Sphere. Journal of Research in Science Teaching, 52 (2), 145-163.

Feinstein N. (2011) Salvaging science literacy. Science education, 95 (1), 168-185.

Fernández-Arroyo J., Rodríguez-Marín F. (2016) Los procesos de enseñanza-aprendizaje relacionados con el agua en el marco de las hipótesis de transición. Revista Eureka sobre Enseñanza y Divulgación de las Ciencias, 14 (1), 227-243.

Fernández-Muerza A. (2004) Estudio del periodismo de información cientifica en la prensa de referencia: el caso español a partir de un análisis comparativo. Tesis doctoral, Universidad del País Vasco. Recuperado el 12/07/13 de http://e-ciencia.com/afm/tesis-alex.pdf.

Fowler S. R., Zeidler D. L. (2016) Lack of Evolution Acceptance Inhibits Students' Negotiation of Biology-based Socioscientific Issues. Journal of Biological Education, 50 (4), $407-424$.

Jarman R., McClune B. (2007) Developing Scientific Literacy. Using News Media in the Classroom. New York: McGraw-Hill.

Jiménez-Liso, M.R.; Jiménez-Liso, M. R., Hernández-Villalobos, L. y Lapetina, J. (2010). Dificultades y propuestas para utilizar las noticias científicas de la prensa en el aula de ciencias. Revista Eureka sobre Enseñanza y Divulgación de las Ciencias, 7(1), 107-126.

Latour B. (2005) Reassembling the social: An introduction to actor-network-theory. Oxford: Clarendon.

McClune B., Jarman R. (2014) Promoting science literacy via science journalism: Issues and challenges. En L. Tan Wee Hin, R. Subramaniam (Eds.), Communicating science to the public, (3-20). Dordrecht: Springer.

NRC (National Research Council) (1996) National Science Education Standards. Washington, DC: National Academy Press.

Poberezshkaya M. (2015) Media coverage of climate change in Russia: Governmental bias and climate science. Public Understanding of Science, 24 (1), 96-111.

Roberts D. A. (2007) Scientific Literacy/Science Literacy. En Abell, S. K. y Lederman, N. G. (Eds.), Handbook research on science education. Mahwah, New Jersey: Lawrence Erlbaum.

Sadler T.D. (2011) Socio-scientific Issues in the Classroom: Teaching, Learning and Research. Netherlands: Springer.

Sadler T.D. (2004) Informal reasoning regarding socioscientific issues: A critical review of research. Journal of Research in Science Teaching, 41, 513-536.

Schäfer M.S. (2012) Taking stock: A meta-analysis of the studies on the Media's coverage of science. Public Understanding of Science, 21(6), 650-663.

Thompson J. B. (1995) The media and modernity. Stanford, CA: Stanford University Press.

Venturini T. (2010) Diving in magma: How to explore controversies with actor-network theory. Public Understanding of Science, 19 (3), 258-253.

Vílchez J. E. (2009) La problemática ambiental en los medios. Propuesta de un protocolo de análisis para su uso como recurso didáctico. Enseñanza de las ciencias, 27 (39), 421-432. 
Zeidler D. L. (2014) Socioscientific Issues as a Curriculum Emphasis: Theory, Research and Practice en Lederman, N.G y Abell, S.K. (Eds.) Handbook of Research on Science Education, II, Routledge, 697-726.

Zeidler D. L., Berkowitz M., Bennett K. (2013) Thinking (scientifically) responsibly: The cultivation of character in a global science education community. En M.P. Mueller, D.J. Tippins, y A.J. Steward (Eds.), Assessing schools for generation R (Responsibility): A guide to legislation and school policy in science education, Netherlands: Springer, 83-99.

Zimmerman C., Bisanz G. L., Bisanz J., Klein J. S., Klein P. (2010) Science at the supermarket: A comparison of what appears in the popular press, experts' advice to readers, and what students want to know. Public Understanding of Science, 10, 37-58. 